\title{
Square of the Error Octonionic Theorem and Hypercomplex Fourier Serier
}

\author{
C.A.P. MARTINEZ ${ }^{1}$, A.L.M. MARTINEZ ${ }^{1}$, \\ M.F. BORGES ${ }^{2}$ and E.V. CASTELANI ${ }^{3 *}$
}

Received on March 21, 2013 / Accepted on October 13, 2013

\begin{abstract}
The focus of this paper is to address some classical results for a class of hypercomplex numbers. More specifically we present an extension of the Square of the Error Theorem and a Bessel inequality for octonions.
\end{abstract}

Keywords: hypercomplex functions, Fourier series, octonions.

\section{INTRODUCTION AND MOTIVATION}

Octonions are hypercomplex numbers and in many ways may be regarded as non-associative quaternions. Nowadays they have been used in appropriated approaches of higher dimensional physics [5, 8], such as M-theory, Strings and alternative gravity theories. In this paper, with the main purpose in the nearest future of making a larger application of hypercomplex in unified field theories, and motivated both by earlier works by Eilenberg, Niven [7], Deavours [6], Sinegre [16], and on recent results obtained by some of the authors $[2,3,4,12,13,14]$, Boek, Gurlebeck [1], and Lam [11], we have concentrated our efforts in constructing an extension of the Algebra of hypercomplex. Hypercomplex numbers are mainly concerned to quaternions and octonions. Their algebras may regarded as extensions of the ordinary bi-dimensional complex algebra $[10,15]$, either being non-commutative and associative for quaternions or non-commutative and non-associative in the octonionic case.

Quaternions were discovered in 1843 by Willian R. Hamilton (op. cit. [16]), and in that same year John T. Graves, a Hamilton's friend, found an 8-dimensional Algebra whose property

\footnotetext{
*Corresponding author: Emerson Vitor Castelani

${ }^{1}$ Coordenação de Matemática, COMAT, UTFPR - Universidade Tecnológica Federal do Paraná, Campus Cornélio Procópio, Av. Alberto Carazzai, 1640, 86300-000 Cornélio Procópio, PR, Brasil. E-mails: crismartinez@utfpr.edu.br; andrelmmartinez@yahoo.com.br

2 Department of Computing, UNESP - State University of São Paulo at São José do Rio Preto, 15054-000 São José do Rio Preto, SP, Brazil. E-mail: borges@ibilce.unesp.br

3 Departamento de Matemática, DMA, UEM - Universidade Estadual de Maringá, Av. Colombo, 5790, 87020-900 Maringá, PR, Brasil. E-mail: emersonvitor@gmail.com
} 
on non-associativity in a multiplication table holds. Further on, two years later, in 1845, after some contributions on this subject by Arthur Cayley octonions have also been named "Cayley numbers".

In the context of the present work, and based in a previous result (op. cit. [13]), we extend for octonions the concept of a 2L-periodical function, that we will call Octonionic Fourier Series. We define an octonionic exponential function, and show both a non-associative expansion of the Moivre Theorem and generalizations of the Euler formula. Finally, we obtain octonionic versions of the Square of the Error Theorem and the Bessel inequality.

\subsection{Hypercomplex Fourier Series}

The octonions are a somewhat nonassociativite extension of the quaternions. They form the 8dimensional normed division algebra on $\mathcal{R}$.

The octonionic algebra, also called octaves denoted for $\mathcal{O}$, is an alternative division algebra.

The octonions set $\mathcal{O}$ is denoted by

$$
\mathcal{O}=\{a, b, c, d, e, f, g, h\} \in \mathcal{R},
$$

where,

$$
\begin{aligned}
(a, b, c, d, e, f, g, h)=\left(a^{\prime}, b^{\prime}, c^{\prime}, d^{\prime}, e^{\prime}, f^{\prime}, g^{\prime}, h^{\prime}\right) \Longleftrightarrow & a=a^{\prime}, b=b^{\prime}, c=c^{\prime}, d=d^{\prime}, \\
& e=e^{\prime}, f=f^{\prime}, g=g^{\prime}, h=h^{\prime} .
\end{aligned}
$$

The octonions do not form a ring due the non-commutativity of the multiplication. Also do not form a group due a nonassociativide of multiplication. They form a Moufang Loop, a Loop with identity element (Jacobson, N., [9]).

Let us consider an octonionic number given by

$$
o=a+b \mathbf{i}+c \mathbf{j}+d \mathbf{k}+e \mathbf{l}+f \mathbf{l i}+g \mathbf{l} \mathbf{j}+h \mathbf{l} \mathbf{k}
$$

The octonion unities $\mathbf{1}, \mathbf{i}, \mathbf{j}, \mathbf{k}, \mathbf{l}, \mathbf{l i}, \mathbf{l j}, \mathbf{l k}$ form an orthonormal base of the 8-dimensional algebra. Similarly to [13] we start our contribution in considering $f$ a function defined on interval $[-L, L], L>0$, and outside of this interval set as $f(x)=f(x+2 L)$, that is, $f(x)$ is $2 L$-periodical. If $f$ and $f^{\prime}$ are piecewise continuous then the series of function given below,

$$
\frac{a_{0}}{2}+\sum_{n=1}^{\infty}\left[a_{n} \cos \left(\frac{n \pi x}{L}\right)+b_{n} \sin \left(\frac{n \pi x}{L}\right)\right]
$$

is convergent and the limit is

$$
\tilde{f}(x)=\frac{\lim _{a \rightarrow x^{+}} f(x)+\lim _{a \rightarrow x^{-}} f(x)}{2} .
$$


The coefficients of Fourier of $f, a_{0}, a_{n}$ and $b_{n}$ given by:

$$
\begin{gathered}
a_{0}=\frac{1}{L} \int_{-L}^{L} f(x) d x, \\
a_{n}=\frac{1}{L} \int_{-L}^{L} f(x) \cos \left(\frac{n \pi x}{L}\right) d x,
\end{gathered}
$$

and

$$
b_{n}=\frac{1}{L} \int_{-L}^{L} f(x) \sin \left(\frac{n \pi x}{L}\right) d x .
$$

The trigonometric series presented in (1.1) with this choice of coefficients is the Fourier series of $f$.

Now, we will detail some properties considering octonions $o \in \mathcal{O}$ given by

$$
o=u_{1}+u_{2} i+u_{3} j+u_{4} k+u_{5} l+u_{6} l i+u_{7} l j+u_{8} l k=u_{1}+\vec{u} .
$$

The extended equation of De Moivre for octonions is given by:

$$
\begin{aligned}
e^{o}= & e^{u_{1}}\left\{\cos \left(\sqrt{u_{2}^{2}+u_{3}^{2}+u_{4}^{2}+u_{5}^{2}+u_{6}^{2}+u_{7}^{2}+u_{8}^{2}}\right)\right. \\
& \left.+\vec{u}\left(\frac{\sin \left(\sqrt{u_{2}^{2}+u_{3}^{2}+u_{4}^{2}+u_{5}^{2}+u_{6}^{2}+u_{7}^{2}+u_{8}^{2}}\right)}{\sqrt{u_{2}^{2}+u_{3}^{2}+u_{4}^{2}+u_{5}^{2}+u_{6}^{2}+u_{7}^{2}+u_{8}^{2}}}\right)\right\}
\end{aligned}
$$

Then, using (1.4) we can obtain:

$$
\frac{e^{i y}+e^{-i y}}{2}=\frac{e^{0}\left(\cos |y|+i \frac{y}{|y|} \sin |y|\right)+e^{0}\left(\cos |-y|+i \frac{-y}{|-y|} \sin |-y|\right)}{2} .
$$

Since $\cos (-y)=\cos (y)$ and $\sin (-y)=-\sin (y)$ we have that

$$
\frac{e^{i y}+e^{-i y}}{2}=\cos (y)
$$

Following the same arguments we can get

$$
\begin{aligned}
\cos y & =\frac{e^{i y}+e^{-i y}}{2}=\frac{e^{j y}+e^{-j y}}{2}=\frac{e^{k y}+e^{-k y}}{2}=\frac{e^{l y}+e^{-l y}}{2} \\
& =\frac{e^{(l i) y}+e^{-(l i) y}}{2}=\frac{e^{(l j) y}+e^{-(l j) y}}{2}=\frac{e^{(l k) y}+e^{-(l k) y}}{2}
\end{aligned}
$$

and

$$
\begin{aligned}
\sin y & =\frac{e^{i y}-e^{-i y}}{2 i}=\frac{e^{j y}-e^{-j y}}{2 j}=\frac{e^{k y}-e^{-k y}}{2 k}=\frac{e^{l y}-e^{-l y}}{2 l} \\
& =\frac{e^{(l i) y}-e^{-(l i) y}}{2 l i}=\frac{e^{(l j) y}-e^{-(l j) y}}{2 l j}=\frac{e^{(l k) y}-e^{-(l k) y}}{2 l k} .
\end{aligned}
$$


From (1.5) and (1.6), we obtain,

$$
\begin{aligned}
& \cos y=\frac{\left(e^{i y}+e^{j y}+\cdots+e^{(l k) y}\right)+\left(e^{-i y}+e^{-j y}+\cdots+e^{-(l k) y}\right)}{14}, \\
& \sin y=\frac{1}{7}\left[\frac{e^{i y}-e^{-i y}}{2 i}+\frac{e^{j y}-e^{-j y}}{2 j}+\cdots+\frac{e^{(l k) y}-e^{-(l k) y}}{2 l k}\right] .
\end{aligned}
$$

Consequently,

$$
\begin{aligned}
& a_{n} \cos \left(\frac{n \pi x}{L}\right)+b_{n} \sin \left(\frac{n \pi x}{L}\right) \\
= & a_{n}\left[\frac{\left(e^{i\left(\frac{n \pi x}{L}\right)}+\ldots+e^{(l k)\left(\frac{n \pi x}{L}\right)}\right)}{14}+\frac{\left(e^{-i\left(\frac{n \pi x}{L}\right)}+\ldots+e^{-(l k)\left(\frac{n \pi x}{L}\right)}\right)}{14}\right] \\
+ & b_{n} \frac{1}{7}\left[\frac{e^{i\left(\frac{n \pi x}{L}\right)}-e^{-i\left(\frac{n \pi x}{L}\right)}}{2 i}+\frac{e^{(l k)\left(\frac{n \pi x}{L}\right)}-e^{-(l k)\left(\frac{n \pi x}{L}\right)}}{2 l k}\right] \\
= & \frac{1}{7}[\underbrace{\left(\frac{a_{n}}{2}+\frac{b_{n}}{2 i}\right)}_{c_{n}^{1}} e^{\frac{i n \pi x}{L}}+\underbrace{\left(\frac{a_{n}}{2}-\frac{b_{n}}{2 i}\right) e^{\frac{-i n \pi x}{L}}+\ldots}_{c_{-n}^{1}}] \\
+ & \underbrace{\left(\frac{a_{n}}{2}+\frac{b_{n}}{2(l k)}\right)}_{c_{n}^{7}} e^{\frac{(l k n n \pi x}{L}}+\underbrace{\left(\frac{a_{n}}{2}-\frac{b_{n}}{2(l k)}\right)}_{c_{-n}^{7}} e^{\frac{-(l k n n \pi x}{L}}] .
\end{aligned}
$$

Noting that

$$
c_{n}^{1}=\frac{a_{n}}{2}+\frac{b_{n}}{2 i}=\frac{1}{2}\left(a_{n}-i b_{n}\right)
$$

and using (1.2) and (1.3) we have:

$$
c_{n}^{1}=\frac{1}{2 L} \int_{-L}^{L} f(x) e^{-i\left(\frac{n \pi x}{L}\right)} d x .
$$

Again, using the same argument we can get:

$$
c_{-n}^{1}=\frac{1}{2 L} \int_{-L}^{L} f(x) e^{-i\left(\frac{(-n) \pi x}{L}\right)} d x .
$$

Extending the ideas used, we conclude that:

$$
c_{n}^{2}=\frac{1}{2 L} \int_{-L}^{L} f(x) e^{-j\left(\frac{n \pi x}{L}\right)} d x,
$$


and

$$
c_{-n}^{2}=\frac{1}{2 L} \int_{-L}^{L} f(x) e^{j\left(\frac{n \pi x}{L}\right)} d x,
$$

since $(j)^{2}=-1$.

$$
c_{n}^{3}=\frac{1}{2 L} \int_{-L}^{L} f(x) e^{-k\left(\frac{n \pi x}{L}\right)} d x
$$

and

$$
c_{-n}^{3}=\frac{1}{2 L} \int_{-L}^{L} f(x) e^{k\left(\frac{n \pi x}{L}\right)} d x
$$

since $(k)^{2}=-1$.

$$
c_{n}^{4}=\frac{1}{2 L} \int_{-L}^{L} f(x) e^{-l\left(\frac{n \pi x}{L}\right)} d x
$$

and

$$
c_{-n}^{4}=\frac{1}{2 L} \int_{-L}^{L} f(x) e^{l\left(\frac{n \pi x}{L}\right)} d x
$$

since $(l)^{2}=-1$.

$$
c_{n}^{5}=\frac{1}{2 L} \int_{-L}^{L} f(x) e^{-l i\left(\frac{n \pi x}{L}\right)} d x
$$

and

$$
c_{-n}^{5}=\frac{1}{2 L} \int_{-L}^{L} f(x) e^{l i\left(\frac{n \pi x}{L}\right)} d x,
$$

since $(l i)^{2}=-1$.

$$
c_{n}^{6}=\frac{1}{2 L} \int_{-L}^{L} f(x) e^{-l j\left(\frac{n \pi x}{L}\right)} d x
$$

and

$$
c_{-n}^{6}=\frac{1}{2 L} \int_{-L}^{L} f(x) e^{l j\left(\frac{n \pi x}{L}\right)} d x
$$

since $(l j)^{2}=-1$.

$$
c_{n}^{7}=\frac{1}{2 L} \int_{-L}^{L} f(x) e^{-l k\left(\frac{n \pi x}{L}\right)} d x,
$$

and

$$
c_{-n}^{7}=\frac{1}{2 L} \int_{-L}^{L} f(x) e^{l k\left(\frac{n \pi x}{L}\right)} d x,
$$

since $(l k)^{2}=-1$.

From (1.7), (1.8), (1.9), (1.10), (1.11), (1.12), (1.13), (1.14),(1.15), (1.16), (1.17), (1.18), (1.19) and (1.20) we can get

$$
\begin{aligned}
& \sum_{n=1}^{\infty}\left[a_{n} \cos \left(\frac{n \pi x}{L}\right)+b_{n} \sin \left(\frac{n \pi x}{L}\right)\right] \\
= & \frac{1}{7} \sum_{-\infty, n \neq 0}^{\infty}\left[c_{n}^{1} e^{\frac{i n \pi x}{L}}+c_{n}^{2} e^{\frac{j n \pi x}{L}}+c_{n}^{3} e^{\frac{k n \pi x}{L}}+c_{n}^{4} e^{\frac{\ln \pi x}{L}}+c_{n}^{5} e^{\frac{\operatorname{lin} \pi x}{L}}+c_{n}^{6} e^{\frac{l j n \pi x}{L}}+c_{n}^{7} e^{\frac{l k n \pi x}{L}}\right]
\end{aligned}
$$


Since $f: R \rightarrow R$ is periodic, with period $2 L, f$ and $f^{\prime}$ are piecewise continuous, we have that the Fourier series of $f$ presented in (1.1) can be written as follows

$$
\begin{aligned}
\tilde{f}(x)= & c_{o}+\frac{1}{7}\left\{\sum_{-\infty, n \neq 0}^{\infty} c_{n}^{1} e^{\frac{i n \pi x}{L}}+c_{n}^{2} e^{\frac{j n \pi x}{L}}+c_{n}^{3} e^{\frac{k n \pi x}{L}}\right. \\
& \left.+c_{n}^{4} e^{\frac{l n \pi x x}{L}}+c_{n}^{5} e^{\frac{l i n \pi x}{L}}+c_{n}^{6} e^{\frac{l j n \pi x}{L}}+c_{n}^{7} e^{\frac{l k n \pi x}{L}}\right\},
\end{aligned}
$$

where considering $c_{0}=\frac{a_{0}}{2}$.

The series (1.21) is the octonionic Fourier series of $f$.

Example 1. Let

$$
f(x)=\left\{\begin{array}{cl}
2, & \text { if } 0 \leq x<\pi \\
0, & \text { if }-\pi<x \leq 0 . \\
f(x+2 \pi)=0 . &
\end{array}\right.
$$

We will determine the octonionic Fourier series of $f$. In fact, calculating the coefficients $c_{0}$, $c_{n}^{1}, c_{n}^{2}, c_{n}^{3}, c_{n}^{4}, c_{n}^{5}, c_{n}^{7}, c_{n}^{7}$ we have

$$
\begin{aligned}
c_{0} & =\frac{a_{0}}{2}=\frac{1}{2 L} \int_{L}^{-L} f(x) d x=\frac{1}{2 \pi}\left[\int_{-\pi}^{0} 0 d x+\int_{0}^{\pi} 2 d x\right]=1, \\
c_{n}^{1} & =\frac{1}{2 \pi} \int_{0}^{\pi} 2 e^{-i n x} d x=\frac{i}{n \pi}[\cos (n \pi)-1]=\frac{i}{n \pi}\left[(-1)^{n}-1\right], \\
c_{n}^{2} & =\frac{1}{2 \pi} \int_{0}^{\pi} 2 e^{-j n x} d x=\frac{j}{n \pi}[\cos (n \pi)-1]=\frac{j}{n \pi}\left[(-1)^{n}-1\right], \\
c_{n}^{3} & =\frac{1}{2 \pi} \int_{0}^{\pi} 2 e^{-k n x} d x=\frac{k}{n \pi}[\cos (n \pi)-1]=\frac{k}{n \pi}\left[(-1)^{n}-1\right], \\
c_{n}^{4} & =\frac{1}{2 \pi} \int_{0}^{\pi} 2 e^{-l n x} d x=\frac{l}{n \pi}[\cos (n \pi)-1]=\frac{l}{n \pi}\left[(-1)^{n}-1\right], \\
c_{n}^{5} & =\frac{1}{2 \pi} \int_{0}^{\pi} 2 e^{-(l i) n x} d x=\frac{(l i)}{n \pi}[\cos (n \pi)-1]=\frac{(l i)}{n \pi}\left[(-1)^{n}-1\right], \\
c_{n}^{6} & =\frac{1}{2 \pi} \int_{0}^{\pi} 2 e^{-(l j) n x} d x=\frac{(l j)}{n \pi}[\cos (n \pi)-1]=\frac{(l j)}{n \pi}\left[(-1)^{n}-1\right], \\
c_{n}^{7} & =\frac{1}{2 \pi} \int_{0}^{\pi} 2 e^{-(l k) n x} d x=\frac{(l k)}{n \pi}[\cos (n \pi)-1]=\frac{(l k)}{n \pi}\left[(-1)^{n}-1\right],
\end{aligned}
$$


thus

$$
\begin{aligned}
\tilde{f}(x)= & 1+\sum_{-\infty, n \neq 0}^{\infty}\left[\frac{i\left[(-1)^{n}-1\right]}{n \pi} e^{i n x}+\frac{j\left[(-1)^{n}-1\right]}{n \pi} e^{j n x}\right. \\
& +\frac{k\left[(-1)^{n}-1\right]}{n \pi} e^{k n x}+\frac{l\left[(-1)^{n}-1\right]}{n \pi} e^{l n x}+\frac{(l i)\left[(-1)^{n}-1\right]}{n \pi} e^{(l i) n x} \\
& \left.+\frac{(l j)\left[(-1)^{n}-1\right]}{n \pi} e^{(l j) n x}+\frac{(l k)\left[(-1)^{n}-1\right]}{n \pi} e^{(l k) n x}\right] .
\end{aligned}
$$

Example 2. Let $f(t)=t$, with $t \in(-1,1)$ and $f(t+2)=f(t)$.

The octonionic coefficients are given by:

$$
\begin{aligned}
c_{0} & =0 \\
c_{n}^{1} & =\frac{1}{2} \int_{-1}^{1} t e^{-i n \pi t} d t=\frac{(-1)^{n+1}}{(i n \pi)}, \\
c_{n}^{2} & =\frac{1}{2} \int_{-1}^{1} t e^{-j n \pi t} d t=\frac{(-1)^{n+1}}{(j n \pi)}, \\
c_{n}^{3} & =\frac{1}{2} \int_{-1}^{1} t e^{-k n \pi t} d t=\frac{(-1)^{n+1}}{(k n \pi)}, \\
c_{n}^{4} & =\frac{1}{2} \int_{-1}^{1} t e^{-\ln \pi t} d t=\frac{(-1)^{n+1}}{(\ln \pi)}, \\
c_{n}^{5} & =\frac{1}{2} \int_{-1}^{1} t e^{-(l i) n \pi t} d t=\frac{(-1)^{n+1}}{((l i) n \pi)}, \\
c_{n}^{6} & =\frac{1}{2} \int_{-1}^{1} t e^{-(l j) n \pi t} d t=\frac{(-1)^{n+1}}{((l j) n \pi)}, \\
c_{n}^{7} & =\frac{1}{2} \int_{-1}^{1} t e^{-(l k) n \pi t} d t=\frac{(-1)^{n+1}}{((l k) n \pi)},
\end{aligned}
$$

Then, the octonionic Fourier series of $f$ is

$$
\begin{aligned}
\tilde{f}(t)= & \sum_{-\infty, n \neq 0}^{\infty}\left[\frac{(-1)^{n+1}}{i n \pi} e^{i n \pi t}+\frac{(-1)^{n+1}}{j n \pi} e^{j n \pi t}\right. \\
& +\frac{(-1)^{n+1}}{k n \pi} e^{k n \pi t}+\frac{(-1)^{n+1}}{\ln \pi} e^{l n \pi t}+\frac{(-1)^{n+1}}{(l i) n \pi} e^{(l i) n \pi t} \\
& \left.+\frac{(-1)^{n+1}}{(l j) n \pi} e^{(l j) n \pi t}+\frac{(-1)^{n+1}}{(l k) n \pi} e^{(l k) n \pi t}\right]
\end{aligned}
$$

Tend. Mat. Apl. Comput., 14, N. 3 (2013) 


\section{AN EXTENSION OF THE SQUARE OF THE ERROR THEOREM FOR OCTONIONIC FOURIER SERIES AND THE BESSEL INEQUALITY}

In this Section we will present an important property of the Hypercomplex Fourier Series derived from some results from the classical Complex Analysis [10]. We also make an extension of the so called Square of the Error Theorem (Walter Rudin [15]).

For the purposes of this section, we denote $\phi$ the octonionic function defined by

$$
\begin{gathered}
\phi: R \rightarrow \mathcal{O}, \\
\phi(x)=\phi_{1}(x)+\phi_{2}(x) i+\phi_{3}(x) j+\phi_{4}(x) k+\phi_{5}(x) l+\phi_{6}(x) l i+\phi_{7}(x) l j+\phi_{8}(x) l k
\end{gathered}
$$

where $\phi_{1}, \phi_{2}, \phi_{3}, \phi_{4}, \phi_{5}, \phi_{6}, \phi_{7}$ and $\phi_{8}$ are real functions.

Thus we can consider

$$
\begin{gathered}
\overline{\phi(x)}=\phi_{1}(x)-\left[\phi_{2}(x) i+\phi_{3}(x) j+\phi_{4}(x) k+\phi_{5}(x) l+\phi_{6}(x) l i+\phi_{7}(x) l j+\phi_{8}(x) l k\right], \\
|\phi(x)|^{2}=\phi(x) \overline{\phi(x)}=\sum_{m=1}^{8}\left(\phi_{m}(x)\right)^{2}
\end{gathered}
$$

Definition 2.1. Let $\left\{\phi_{n}\right\}, n=1,2,3,4, \ldots$ be a sequence of octonionic functions on $[a, b]$, such that

$$
\int_{a}^{b} \phi_{n}(x) \overline{\phi_{m}(x)} d x=0, \quad(n \neq m) .
$$

Then, we say that $\left\{\phi_{n}\right\}$ is an orthogonal system of octonionic functions on $[a, b]$. In addition, if

$$
\int_{a}^{b}\left|\phi_{n}(x)\right|^{2} d x=1,
$$

for all $n$, we say that $\left\{\phi_{n}\right\}$ is orthonormal. Here are some examples.

Example 3. The sequence of functions

$$
\begin{aligned}
\phi_{n}^{1}(x) & =\frac{1}{\sqrt{2 L}} e^{\frac{i n \pi x}{L}}, n=1,2,3, \ldots \\
\phi_{n}^{2}(x) & =\frac{1}{\sqrt{2 L}} e^{\frac{i n \pi x}{L}}, n=1,2,3, \ldots \\
\phi_{n}^{3}(x) & =\frac{1}{\sqrt{2 L}} e^{\frac{k n \pi x}{L}}, n=1,2,3, \ldots \\
\phi_{n}^{4}(x) & =\frac{1}{\sqrt{2 L}} e^{\frac{\ln \pi x}{L}}, n=1,2,3, \ldots \\
\phi_{n}^{5}(x) & =\frac{1}{\sqrt{2 L}} e^{\frac{\operatorname{lin} \pi x}{L}}, n=1,2,3, \ldots
\end{aligned}
$$




$$
\begin{aligned}
& \phi_{n}^{6}(x)=\frac{1}{\sqrt{2 L}} e^{\frac{l j n \pi x}{L}}, n=1,2,3, \ldots \\
& \phi_{n}^{7}(x)=\frac{1}{\sqrt{2 L}} e^{\frac{l k n \pi x}{L}}, n=1,2,3, \ldots
\end{aligned}
$$

form orthonormal system on $[-L, L]$.

Example 4. The sequence of functions

$$
\begin{aligned}
& \phi_{n}(x)=\frac{1}{\sqrt{56 L}}\left(e^{\frac{i n \pi x}{L}}+e^{\frac{j n \pi x}{L}}+e^{\frac{k n \pi x}{L}}+e^{\frac{\ln \pi x x}{L}}+e^{\frac{l i n \pi x}{L}}+e^{\frac{l i n \pi \pi x}{L}}+e^{\frac{l k n \pi x}{L}}\right), \\
& \text { for } n=1,2,3, \ldots \text {. }
\end{aligned}
$$

form orthonormal system on $[-L, L]$.

Motivated by example 4, we define

$$
\begin{aligned}
& c_{n}^{1}+c_{n}^{2} i+c_{n}^{3} j+c_{n}^{4} k+c_{n}^{5} l+c_{n}^{6} l i+c_{n}^{7} l j+c_{n}^{8} l k \\
= & \int_{a}^{b} f(t) \overline{\phi_{n}(t)} d t, \text { for all } n=1,2,3, \ldots,
\end{aligned}
$$

where $\left\{\phi_{n}\right\}$ is an orthonormal sequence in $[a, b]$ and $c_{n}^{1}, c_{n}^{2}, c_{n}^{3}, c_{n}^{4}, c_{n}^{5}, c_{n}^{6}, c_{n}^{7}$ and $c_{n}^{8}$ are real sequences.

We call $c_{n}$ the $n$-th octonionic Fourier coefficient of $f$ (relative to $\left\{\phi_{n}\right\}$ ). We write

$$
\sum_{1}^{\infty}\left(c_{n}^{1}+c_{n}^{2} i+c_{n}^{3} j+c_{n}^{4} k+c_{n}^{5} l+c_{n}^{6} l i+c_{n}^{7} l j+c_{n}^{8} l k\right) \phi_{n}(x)
$$

and call this series the octonionic Fourier series of $f$.

The following theorem extends some classical results (op. cit. [15]), which have already been extended for the quaternionic case (op. cit. [13]). More specifically we show that partial sums of octonionic Fourier Series of a function $f$, have certain minimum property. Let us assume that $f$ is a real function.

Theorem 2.1. Let $\left\{\phi_{n}\right\}$ be orthonormal sequence of octonionic functions on $[a, b]$. Consider the $n$-th partial sum of the octonionic Fourier series of $f$

$$
s_{n}(x)=\sum_{m=1}^{n}\left(c_{m}^{1}+c_{m}^{2} i+c_{m}^{3} j+c_{m}^{4} k+c_{m}^{5} l+c_{m}^{6} l i+c_{m}^{7} l j+c_{m}^{8} l k\right) \phi_{m}(x),
$$

where $c_{m}^{1}, c_{m}^{2}, c_{m}^{3}, c_{m}^{4}, c_{m}^{5}, c_{m}^{6}, c_{m}^{7}$ and $c_{m}^{8}$ are given in (2.3). In addition, define

$$
t_{n}(x)=\sum_{m=1}^{n}\left(d_{m}^{1}+d_{m}^{2} i+d_{m}^{3} j+d_{m}^{4} k+d_{m}^{5} l+d_{m}^{6} l i+d_{m}^{7} l j+d_{m}^{8} l k\right) \phi_{m}(x),
$$


where $d_{m}^{1}, d_{m}^{2}, d_{m}^{3}, d_{m}^{4}, d_{m}^{5}, d_{m}^{6}, d_{m}^{7}$ and $d_{m}^{8}$ are real sequences. Then

$$
\int_{a}^{b}\left|f(x)-s_{n}(x)\right|^{2} d x \leq \int_{a}^{b}\left|f(x)-t_{n}(x)\right|^{2} d x,
$$

and equality holds if and only if

$$
\begin{aligned}
& d_{m}^{1}=c_{m}^{1}, \quad d_{m}^{2}=c_{m}^{2}, \quad d_{m}^{3}=c_{m}^{3}, \quad d_{m}^{4}=c_{m}^{4}, \quad d_{m}^{5}=c_{m}^{5}, \\
& d_{m}^{6}=c_{m}^{6}, \quad d_{m}^{7}=c_{m}^{7} \text { and } d_{m}^{8}=c_{m}^{8}(m=1,2, \ldots, n) .
\end{aligned}
$$

That means, among all functions $t_{n}, s_{n}$ gives the best possible mean square approximation to $f$.

Proof. To simplify the notation, let $\int$ be the integral over $[a, b]$ and $\sum$ the sum from 1 to $n$. From the definition of (2.6) and (2.3), we have

$$
\begin{aligned}
\int f \overline{t_{n}} & =\int f\left[\overline{\sum\left(d_{m}^{1}+d_{m}^{2} i+d_{m}^{3} j+d_{m}^{4} k+d_{m}^{5} l+d_{m}^{6} l i+d_{m}^{7} l j+d_{m}^{8} l k\right) \phi_{m}}\right] \\
& =\sum \overline{\left(d_{m}^{1}+d_{m}^{2} i+d_{m}^{3} j+d_{m}^{4} k+d_{m}^{5} l+d_{m}^{6} l i+d_{m}^{7} l j+d_{m}^{8} l k\right)} \int f \overline{\phi_{m}} \\
& =\sum \overline{\left(d_{m}^{1}+d_{m}^{2} i+\ldots+d_{m}^{7} l j+d_{m}^{8} l k\right)}\left(c_{m}^{1}+c_{m}^{2} i+\ldots+c_{m}^{7} l j+c_{m}^{8} l k\right) .
\end{aligned}
$$

Consequently,

$$
\begin{aligned}
\int f \overline{t_{n}}+\int \bar{f} t_{n}= & \sum\left[2\left(d_{m}^{1} c_{m}^{1}\right)+2\left(d_{m}^{2} c_{m}^{2}\right)+2\left(d_{m}^{3} c_{m}^{3}\right)+2\left(d_{m}^{4} c_{m}^{4}\right)\right. \\
& \left.+2\left(d_{m}^{5} c_{m}^{5}\right)+2\left(d_{m}^{6} c_{m}^{6}\right)+2\left(d_{m}^{7} c_{m}^{7}\right)+2\left(d_{m}^{8} c_{m}^{8}\right)\right] .
\end{aligned}
$$

Furthermore,

$$
\begin{aligned}
\int\left|t_{n}\right|^{2} & =\int t_{n} \overline{t_{n}} \\
& =\int \sum_{m=1}^{n}\left[\left(d_{m}^{1}+\ldots+d_{m}^{8} l k\right) \phi_{m}\right] \overline{\sum_{p=1}^{n}\left[\left(d_{p}^{1}+\ldots+d_{p}^{8} l k\right) \phi_{p}\right]} \\
& =\sum_{m=1}^{n}\left[\left(d_{m}^{1}\right)^{2}+\left(d_{m}^{2}\right)^{2}+\left(d_{m}^{3}\right)^{2}+\left(d_{m}^{4}\right)^{2}+\left(d_{m}^{5}\right)^{2}+\left(d_{m}^{6}\right)^{2}+\left(d_{m}^{7}\right)^{2}+\left(d_{m}^{8}\right)^{2}\right]
\end{aligned}
$$

since $\left\{\phi_{n}\right\}$ is orthonormal. Therefore,

$$
\begin{aligned}
\int\left|f-t_{n}\right|^{2} & =\int|f|^{2}-\int f \overline{t_{n}}-\int \bar{f} t_{n}+\int\left|t_{n}\right|^{2} \\
& =\int|f|^{2}-\sum\left[2\left(d_{m}^{1} c_{m}^{1}\right)+2\left(d_{m}^{2} c_{m}^{2}\right)+\ldots+2\left(d_{m}^{7} c_{m}^{7}\right)+2\left(d_{m}^{8} c_{m}^{8}\right)\right]
\end{aligned}
$$




$$
\begin{aligned}
& +\sum_{m=1}^{n}\left[\left(d_{m}^{1}\right)^{2}+\left(d_{m}^{2}\right)^{2}+\ldots+\left(d_{m}^{7}\right)^{2}+\left(d_{m}^{8}\right)^{2}\right] \\
= & \int|f|^{2}+\sum\left[\left(d_{m}^{1}-c_{m}^{1}\right)^{2}+\left(d_{m}^{2}-c_{m}^{2}\right)^{2}+\ldots+\left(d_{m}^{7}-c_{m}^{7}\right)^{2}\right. \\
& \left.+\left(d_{m}^{8}-c_{m}^{8}\right)^{2}\right]-\sum_{m=1}^{n}\left[\left(c_{m}^{1}\right)^{2}+\left(c_{m}^{2}\right)^{2}+\ldots+\left(c_{m}^{7}\right)^{2}+\left(c_{m}^{8}\right)^{2}\right]
\end{aligned}
$$

which is minimized if and only if

$$
\begin{aligned}
& d_{m}^{1}=c_{m}^{1}, \quad d_{m}^{2}=c_{m}^{2}, \quad d_{m}^{3}=c_{m}^{3}, \quad d_{m}^{4}=c_{m}^{4}, \quad d_{m}^{5}=c_{m}^{5}, \\
& d_{m}^{6}=c_{m}^{6}, \quad d_{m}^{7}=c_{m}^{7} \text { and } d_{m}^{8}=c_{m}^{8}(m=1,2, \ldots, n) .
\end{aligned}
$$

Corollary 2.1. Let $\left\{\phi_{n}\right\}$ be orthonormal sequence of octonionic functions on $[a, b]$, and if

$$
f(x) \sim \sum_{m=1}^{\infty}\left(c_{m}^{1}+c_{m}^{2} i+c_{m}^{3} j+c_{m}^{4} k+c_{m}^{5} l+c_{m}^{6} l i+c_{m}^{7} l j+c_{m}^{8} l k\right) \phi_{m}(x)
$$

then

$$
\sum_{m=1}^{\infty}\left[\left(c_{m}^{1}\right)^{2}+\left(c_{m}^{2}\right)^{2}+\ldots+\left(c_{m}^{7}\right)^{2}+\left(c_{m}^{8}\right)^{2}\right] \leq \int_{a}^{b}|f(x)|^{2} d x
$$

Proof. In the proof of Theorem (2.1) we found

$$
\begin{aligned}
\int\left|f-t_{n}\right|^{2}= & \int|f|^{2}+\sum\left[\left(d_{m}^{1}-c_{m}^{1}\right)^{2}+\left(d_{m}^{2}-c_{m}^{2}\right)^{2}+\left(d_{m}^{7}-c_{m}^{7}\right)^{2}+\left(d_{m}^{8}-c_{m}^{8}\right)^{2}\right] \\
& -\sum_{m=1}^{n}\left[\left(c_{m}^{1}\right)^{2}+\left(c_{m}^{2}\right)^{2}+\ldots+\left(c_{m}^{7}\right)^{2}+\left(c_{m}^{8}\right)^{2}\right]
\end{aligned}
$$

putting $d_{m}^{1}=c_{m}^{1}, d_{m}^{2}=c_{m}^{2}, d_{m}^{3}=c_{m}^{3}, d_{m}^{4}=c_{m}^{4}, d_{m}^{5}=c_{m}^{5}, d_{m}^{6}=c_{m}^{6}, d_{m}^{7}=c_{m}^{7}$ and $d_{m}^{8}=c_{m}^{8}$ in this calculation, we obtain

$$
\sum_{m=1}^{n}\left[\left(c_{m}^{1}\right)^{2}+\left(c_{m}^{2}\right)^{2}+\ldots+\left(c_{m}^{7}\right)^{2}+\left(c_{m}^{8}\right)^{2}\right] \leq \int_{a}^{b}|f(x)|^{2} d x,
$$

since $\int\left|f-t_{n}\right|^{2} \geq 0$. Letting $n \rightarrow \infty$ in (2.10), we obtain (2.9), this inequality is a generalization for octonions of Bessel.

\section{CONCLUDING REMARKS}

In this paper we have discussed on an extended version of Fourier Series for octonions. Fourier Series are remarkable in the way through an orthonormal basis they approximate functions associated to physical problems such as conducting heat and vibrations. 
Furthermore, as not few models of Theoretical Physics may be analysed through the geometry and algebra of hypercomplex, it will be our concern to concentrate the next steps in making all possible applications of our results in the context of unified physical theories for higher dimensional space-times.

RESUMO. O foco deste trabalho é abordar alguns resultados clássicos para uma certa classe de números hipercomplexos. Mais específicamente, apresentamos uma extensão do Teorema do Erro Quadrático e da Desigualdade de Bessel para octônios.

Palavras-chave: Funções hipercomplexas, Séries de Fourier, octônios.

\section{REFERENCES}

[1] S. Bock \& K. Gurlebeck. On a generalized Appell system and monogenic power series. Math. Methods Appl. Sci., 33(4) (2010), 395-411.

[2] M.F. Borges \& J.A. Marão. Geometrical Wave Equation and the Cauchy-like Theorem for Octonions. International Journal of Pure and Applied Mathematics, 79 (2012), 426-459.

[3] M.F. Borges, J.A. Marão \& R.C. Barreiro. A Cauchy-Like Theorem fo Hypercomplex Functions. Journal of Geometry and Topology, 3 (2009), 263-271.

[4] M.F. Borges \& L.F Benzatti. Quasi-conformal Mappping in Octonionic Algebra: A grafcal and anlytical comparison. Far East Journal of Mathematical Sciences, 33 (2009), 355-361.

[5] C. Castro. On the non-commutative and non-associative geometry of octonionic space-time, modified dispersion relations and gran unifications. Journal of Mathematical Physics, 48 (2007), 073517.

[6] C.A. Deavours. The Quaternionic Calculus. The American Mathematical Montly, 80 (1973), 9951008 .

[7] S. Eilenberg \& I. Niven. The Fundamental Theorem of Algebra for quaternioins. Bull. Amer. Math. Society, 50 (1944), 246-248.

[8] F. Gursey \& C. Hsiung. On the role of division, Jordan and Related Algebras in Particle Physics. World Scientific, Singapore (1996).

[9] N. Jacobson. Basic Algebra I, (W.H. Freeman and Company, New York, 1974).

[10] K. Kodairo. Complex Analysis, (Cambrige University Press, Cambrige, 2007).

[11] T.Y. Lam. Handbook of Algebra, 3, (North-Holland, Amsterdam, 2003, 429-454).

[12] J.M. Machado, A.C. de Oliveira, C.A. Pendeza \& M.F. Borges. De Moivre Extended Equation for Octonions and Power Series. International Journal of Pure and Applied Mathematics, 45 (2008), 165-170.

[13] C.A.P. Martinez, M.F. Borges, A.L.M. Martinez \& E.V. Castelani. Fourier Series for Quaternions and the Extension of the Square of the Error Theorem. International Journal of Applied Mathematics, 25 (2012), 559-570.

[14] J. Marão \& M.F. Borges. Liouville's Theorem and Power Series for Quaternionic Functions. International Journal of Pure and Applied Mathematics, 71 (2011), 383-389. 
[15] W. Rudin. Principles of Mathematical Analysis. Third Ed., (MacGraw-Hill, N. York, 1976).

[16] L. Sinegre. Quaternions and the motion of a solid body about a fixed point according to Hamilton. Rev.-Historie-Math., 1 (1995), 83-109. 